\title{
Oscillation Amplitude Growth for a Decelerating Object with Constant Pitch Damping
}

\author{
Mark Schoenenberger* \\ Eric M. Queen ${ }^{\dagger}$ \\ NASA Langley Research Center, Hampton, VA 23681 \\ and \\ Daniel Litton $\ddagger$ \\ National Institute of Aerospace, Hampton, VA
}

\begin{abstract}
The equations governing the deceleration and oscillation of a blunt body moving along a planar trajectory are re-expressed in the form of the Euler-Cauchy equation. An analytic solution of this equation describes the oscillation amplitude growth and frequency dilation with time for a statically stable decelerating body with constant pitch damping. The oscillation histories for several constant pitch damping values, predicted by the solution of the Euler-Cauchy equation are compared to POST six degree-of-freedom (6-DoF) trajectory simulations. The simulations use simplified aerodynamic coefficients matching the Euler-Cauchy approximations. Agreement between the model predictions and simulation results are excellent. Euler-Cauchy curves are also fit through nonlinear 6-DoF simulations and ballistic range data to identify static stability and pitch damping coefficients. The model is shown to closely fit through the data points and capture the behavior of the blunt body observed in simulation and experiment. The extracted coefficients are in reasonable agreement with higher fidelity, nonlinear parameter identification results. Finally, a nondimensional version of the Euler-Cauchy equation is presented and shown to be a simple and effective tool for designing dynamically scaled experiments for decelerating blunt capsule flight.
\end{abstract}

\section{Nomenclature}

$\begin{array}{llll}A & \text { Angle-of-attack constant } & N_{I} & \text { Ratio of test } I \text { to full-scale } I \\ C_{A} & \text { Axial force coefficient } & N_{m} & \text { Ratio of test } m \text { to full-scale } m \\ C_{D} & \text { Drag force coefficient } & N_{\rho} & \text { Ratio of test } \rho \text { to full-scale } \rho \\ C_{L} & \text { Lift force coefficient } & S & \text { Reference area }=\frac{\pi}{4} d^{2} \\ C_{m} & \text { Pitching moment coefficient } & s & \text { Speed of sound } \\ C_{m_{q}}+C_{m_{\dot{\alpha}}} & \text { Pitch damping coefficient } & t & \text { Time } \\ C_{N} & \text { Normal force coefficient } & \text { Greek } & \\ d & \text { Diameter } & \alpha & \text { Angle-of-attack } \\ F_{x} & \text { Dimensional axial force } & \gamma & \text { Flight path angle } \\ I & \text { Moment of inertia } & \delta & \text { Phase shift constant } \\ M & \text { Mach number } & \theta & \text { Pitch angle } \\ m & \text { mass } & \mu & \text { Euler-Cauchy damping constant } \\ N_{d} & \text { Ratio of test model } d \text { to full-scale } d & \nu & \text { Euler-Cauchy frequency constant }\end{array}$

*Aerospace Engineer, Exploration Systems Engineering Branch, Member AIAA

${ }^{\dagger}$ Aerospace Engineer, Exploration Systems Engineering Branch, Member AIAA

${ }^{\ddagger}$ Aerospace Engineer, Exploration Systems Engineering Branch, Member AIAA 


Greek (cont'd)
$\xi$
$\rho$
$\psi$
$\omega$

\author{
Linear damping coefficient \\ Density \\ Ballistic range yaw angle \\ Oscillation frequency $(\mathrm{rad} / \mathrm{s})$
}

Subscripts

$\begin{array}{ll}1,2 & \text { Time indices } \\ f & \text { Final conditions } \\ i & \text { Initial conditions } \\ o & \text { Constant velocity }\end{array}$

\section{Introduction}

Ballistic range testing is a useful tool for identifying the the dynamic aerodynamic characteristics of blunt bodies and other projectiles. This test technique has the benefit of testing free flying models with no sting effects, often at greater Reynolds numbers than are possible with wind tunnels. However, the data reduction of ballistic range test results is more complicated and time consuming than forced oscillation wind tunnel techniques. To obtain accurate damping derivatives, expert analysts can take months fitting trajectory simulations through the relatively few data points. Often, it is difficult to get even qualitative information without this detailed analysis, due to the rapid variation of several parameters over the course of a ballistic range shot. Blunt bodies in a ballistic range test decelerate rapidly, so dynamic pressure falls rapidly. This drop in dynamic pressure results in an increase in oscillation amplitude that is on the same order as the growth or decay due to the dynamic stability of a body. Therefore, it is very difficult to infer any information about the dynamic stability of a model from raw ballistic range data. For the quick interpretation of ballistic range data as well as for designing ballistic range test matrices (the set of initial velocities and oscillation amplitudes), it is desirable to have a simple model that captures oscillation growth due to deceleration and dynamic stability, as well as the dilation of the oscillation frequency due to decreasing dynamic pressure. Chapman and Yates ${ }^{1}$ described how blunt capsules with nonlinear dynamic instabilities (undamped at low angles and damped at large angles) will reach a limit cycle, oscillating at an amplitude where the damped and undamped attitudes are in equilibrium. However, for a rapidly decelerating object, the dynamic pressure decrease masks this equilibrium and it is difficult to identify such a limit cycle without detailed nonlinear analysis.

The model presented here predicts the oscillation amplitude growth of a decelerating body. An example of this predictive capability is presented for a typical blunt-body, ballistic-range test case, demonstrating the sensitivity to pitch damping values. These model predictions are compared to 6-DoF trajectory simulations that use simplified aerodynamics, matching the approximations made to develop the analytic solution. Then, the model is fit through a $6-\mathrm{DoF}$ trajectory simulation of the same scenario showing that the behavior predicted by the simple model is in excellent agreement with a higher fidelity simulation using nonlinear aerodynamics. The model is also used to extract aerodynamic coefficients by fitting the Euler-Cauchy curve through raw ballistic range data collected for the Mars Exploration Rover (MER) entry capsule. Finally, the governing equations are nondimensionalized to illustrate how matching ratios of a few key parameters with the same ratios at flight conditions will permit the testing of dynamically scaled models that behave very similarly to full-scale vehicles. The model proves to be useful in predicting oscillation behavior even for trajectories that, in general, violate the assumptions that were required for an analytic solution.

\section{Equations of Motion}

The planar equations of motion for a planetary probe or ballistic range model are given by Equations 1 through 3. Equation 1 describes the sum of the forces acting on the body. Equation 2 describes the change in flight path angle, $\gamma$, due to lift, gravity and centrifugal forces as the body curves around a planet. Equation 3 describes the rotational accelerations due to the sum of the moments, static and dynamic, acting on the vehicle. These equations are valid for a low lift-to-drag vehicle at small angles-of-attack ( $\alpha$ approximately less than $30^{\circ}$ ). It is assumed that lift and pitching moment vary linearly with angle-of-attack, drag is constant with angle-of-attack and all aerodynamic coefficients are invariant with velocity/Mach number.

$$
\dot{V}=-\frac{\rho V^{2} S C_{D}}{2 m}-g \sin \gamma_{o}
$$




$$
\begin{gathered}
\dot{\gamma}=\frac{\rho V S C_{L}}{2 m}-\left(\frac{g}{V}-\frac{V}{R}\right) \cos \gamma_{o} \\
\ddot{\theta}=\frac{\rho V^{2} S d}{2 I}\left(C_{m_{q}} \frac{\dot{\theta} d}{2 V}+C_{m_{\dot{\alpha}}} \frac{\dot{\alpha} d}{2 V}+C_{m_{\alpha}} \alpha\right)
\end{gathered}
$$

Where

$$
\theta=\alpha+\gamma
$$

From these equations, Chapman and Yates ${ }^{1}$ derived a moment equation describing the oscillation amplitude growth of a blunt capsule traveling in a plane defined by the velocity and gravity vectors with distance as the independent variable. The equations developed here will keep time as the independent variable. Chapman and Yates made several simplifying assumptions valid for ballistic range testing (and segments of planetary entry) that will again be made in this analysis. These assumptions will be noted as the equations are developed. First, it is assumed the vehicle is far from terminal velocity or is traveling at a small flight path angle. This means the gravity term in Equation 1 is small and may be neglected. The second derivative of Equation 4 with respect to time is now substituted into Equation 3, using the first derivative of Equation 2 for $\ddot{\gamma}$. The gravity turn and centrifugal acceleration contributions to $\ddot{\gamma}$ are small compared to the contribution due to lift and are therefore neglected. Equation 3 becomes:

$$
\ddot{\alpha}+\left(\frac{\rho V S}{2 m}\right)^{2} C_{D} C_{L_{\alpha}} \alpha+\frac{\rho V S}{2 m} C_{L_{\alpha}} \dot{\alpha}=\frac{\rho V^{2} S d}{2 I}\left(C_{m_{q}} \frac{\dot{\theta} d}{2 V}+C_{m_{\dot{\alpha}}} \frac{\dot{\alpha} d}{2 V}+C_{m_{\alpha}} \alpha\right)
$$

The second term on the LHS of Equation 5, with the assumption of linear variation of $C_{L}$ with $\alpha$, modifies the frequency of oscillation of the system slightly, but is small in this application and can be neglected. The pitch rate, $\dot{\theta}$, can be expressed in terms of $\dot{\alpha}$ and $\dot{\gamma}$ using the first derivative of Equation 4 . The first term on the RHS of Equation 5 is then:

$$
C_{m_{q}} \frac{\dot{\theta} d}{2 V}=C_{m_{q}}\left(\frac{\dot{\alpha} d}{2 V}+\frac{\rho S d C_{L}}{4 m}+\frac{1}{2}\left(\frac{g}{V^{2}}-\frac{d}{R}\right) \cos \gamma_{o}\right)
$$

All of the $\dot{\gamma}$ terms in Equation 6 are small and may be neglected as well. Equation 5 then becomes:

$$
\ddot{\alpha}-\frac{\rho V S}{2 m}\left(-C_{L_{\alpha}}+\frac{m d^{2}}{2 I}\left(C_{m_{q}}+C_{m_{\dot{\alpha}}}\right)\right) \dot{\alpha}-\frac{\rho V^{2} S d}{2 I} C_{m_{\alpha}} \alpha=0
$$

Finally, the following small angle-of-attack, low lift-to-drag $\left(C_{N} \ll C_{A}\right)$ approximations are made:

$$
\left.\begin{array}{c}
C_{D}=C_{A} \cos \alpha+C_{N} \sin \alpha \approx C_{A} \cos \alpha \approx C_{A} \\
C_{L}=C_{N} \cos \alpha-C_{A} \sin \alpha \approx-C_{A} \alpha \\
C_{L_{\alpha}} \approx-C_{A}
\end{array}\right\}
$$

and Equation 7 becomes:

$$
\ddot{\alpha}-\frac{\rho V S}{2 m}\left(C_{A}+\frac{m d^{2}}{2 I}\left(C_{m_{q}}+C_{m_{\dot{\alpha}}}\right)\right) \dot{\alpha}-\frac{\rho V^{2} S d}{2 I} C_{m_{\alpha}} \alpha=0
$$

Equation 9 is of the same form as that developed by Chapman and Yates, expressed as a function of time rather than distance. Assuming a constant velocity over the solution domain, this Equation is of the form:

$$
\ddot{\alpha}+2 \xi \alpha+\omega_{o}^{2} \alpha=0
$$

and has a solution of the form:

$$
\alpha=A e^{-\xi t} \cos \left(\omega_{o} t+\delta\right)
$$

The damping exponent, $\xi$, in this equation is: 


$$
\xi=-\frac{\rho V S}{4 m}\left(C_{A}+\left(C_{m_{q}}+C_{m_{\dot{\alpha}}}\right) \frac{m d^{2}}{2 I}\right)
$$

For blunt body problems, the coefficient of the damping term is much smaller than that of the static moment term $\left(\xi^{2} \ll \omega_{o}^{2}\right)$. Therefore, the frequency of oscillation, $\omega_{o}$, is:

$$
\omega_{o}=\sqrt{-\frac{\rho V^{2} S d}{2 I} C_{m_{\alpha}}}
$$

The constants, $A$ and $\delta$ are determined from boundary conditions of a given problem.

Equation 11 predicts that a blunt body will oscillate at a frequency proportional to the square root of the freestream dynamic pressure and static stability, and inversely proportional to the square root of the body's moment-of-inertia about the pitch axis. The equation also predicts that the angle-of-attack of a body grows or decays exponentially, due to its dynamic stability (for a constant velocity assumption, the $C_{A}$ effect on damping should be neglected). Now, as this solution is for a constant velocity, it is not adequate for predicting or interpreting the behavior in a ballistic range. Accounting for the capsule deceleration, the functional form of the solution of the moment equation changes from that of Equation 11. An equation appropriate for a decelerating body will be developed here. Assuming a constant drag force and freestream density, the time to slow from one velocity to another is found by integrating the axial force over the time interval. For a body that is decelerating significantly or where the gravity vector is not closely aligned with the direction of travel, the axial force equation simply equates the aerodynamic forces with the deceleration of the body:

$$
\begin{gathered}
F_{x}=m \frac{d V}{d t}=\frac{1}{2} \rho V^{2} S C_{D} \approx \frac{1}{2} \rho V^{2} S C_{A} \\
\int_{0}^{t} d t=\frac{2 m}{\rho S C_{A}} \int_{V_{i}}^{V} \frac{d V}{V^{2}} \\
t=\frac{2 m}{\rho S C_{A}}\left(\frac{1}{V}-\frac{1}{V_{i}}\right)
\end{gathered}
$$

For times long after the initial conditions and assuming the initial velocity was very large,

$$
\frac{1}{V_{i}} \ll \frac{1}{V}
$$

Equation 16 shows velocity to vary proportionally to the inverse of time:

$$
V=\frac{2 m}{\rho S C_{A} t}
$$

Substituting this expression for velocity into Equation 9 yields:

$$
t^{2} \ddot{\alpha}-\left(1+\frac{m d^{2}}{2 I} \frac{\left(C_{m_{q}}+C_{m_{\dot{\alpha}}}\right)}{C_{A}}\right) t \dot{\alpha}-\frac{2 m^{2} d C_{m_{\alpha}}}{\rho S I C_{A}^{2}} \alpha=0
$$

Equation 19 is of the form:

$$
t^{2} \ddot{\alpha}+2 \xi t \dot{\alpha}+\omega^{2} \alpha=0
$$

This is the Euler-Cauchy equation. ${ }^{2}$ For the aerodynamic coefficients and mass properties of blunt capsules, the characteristic equation of Equation 20 has complex conjugate roots. Therefore this differential equation has a solution of the form:

$$
\alpha=A t^{\mu} \cos (\nu \ln t+\delta)
$$

Where $A$ is a constant determined by the angle-of-attack oscillation amplitude at the boundary conditions and the constant $\delta$ is a phase lag determined from the boundary conditions. The coefficients $\mu$ and $\nu$ are determined by solving for the roots of the characteristic equation of Equation 20. The coefficient, $\mu$ is: 


$$
\mu=\frac{m d^{2}\left(C_{m_{q}}+C_{m_{\dot{\alpha}}}\right)}{4 I C_{A}}+1
$$

For a body with $C_{m_{q}}+C_{m_{\dot{\alpha}}}=0$, oscillations will grow in direct proportion to time $\left(\alpha_{A m p} \propto t\right)$ as velocity decreases logarithmically. The $C_{A}$ contribution (an approximation of $-C_{L_{\alpha}}$ ) to the damping term in Equation 9 causes oscillation amplitude growth even without a contribution from the capsule dynamic stability coefficient.

The coefficient $\nu$ is :

$$
\nu=\sqrt{\mu^{2}-\frac{8 m^{2} C_{m_{\alpha}}}{\pi \rho d I C_{A}^{2}}}
$$

As with the constant velocity version, for the aerodynamics of typical blunt bodies, the damping term in Equation 20 is very small compared to the static stability term $\left(\xi^{2} \ll \omega^{2}\right)$. Equation 23 then becomes:

$$
\nu=\sqrt{-\frac{8 m^{2} C_{m_{\alpha}}}{\pi \rho d I C_{A}^{2}}}=\sqrt{-\frac{2 m^{2} d C_{m_{\alpha}}}{\rho S C_{A}^{2} I}}
$$

This coefficient, $\nu$ is a frequency of oscillation, multiplied by the natural log of time rather than simply time as in Equation 11. At first glance, the frequency does not look to have the same dependence on dynamic pressure as the constant velocity frequency, $\omega_{o}$ (Equation 13). However, the coefficient, $\nu$, can be re-expressed to provide a more intuitive comparison with this constant velocity frequency. For this discussion, a constant reference velocity, $V_{o}$, is introduced that corresponds to the constant velocity frequency, $\omega_{o}$. For a decelerating body let this reference velocity also correspond to the velocity at which the body is traveling at a particular time, $t_{o}$.

Recalling the relation for velocity (Equation 18), here set to the reference velocity, $V_{o}$, at time, $t_{o}$, we have:

$$
V_{o}^{2} t_{o}^{2}=\frac{4 m^{2}}{\rho^{2} S^{2} C_{A}^{2}}
$$

Substituting this version of the velocity equation into Equation 24 yields:

$$
\nu=\sqrt{-\frac{\rho V_{o}^{2} S d C_{m_{\alpha}}}{2 I} t_{o}^{2}}=\omega_{o} t_{o}
$$

The Euler-Cauchy solution then becomes:

$$
\alpha=A t^{\mu} \cos \left(\omega_{o} t_{o} \ln t+\delta\right)
$$

Now, a comparison of the oscillation frequencies of a constant velocity body at $V_{o}$ and a decelerating body as it passes through $V_{o}$ can be made. To simplify the frequency comparison, it is assumed that the damping for each system is such that oscillation amplitude is constant with time $(\xi=0$, and $\mu=0)$. With this simplification, the second derivatives of equations 11 and 26 divided by the original equations yield the square of the oscillation frequencies. For Equation 11 with no damping, we have:

$$
\sqrt{-\frac{\ddot{\alpha}}{\alpha}}=\omega_{o}
$$

and for Equation 26 with no damping :

$$
\sqrt{-\frac{\ddot{\alpha}}{\alpha}}=\omega_{o} \frac{t_{o}}{t}
$$

Note that Equations 27 and 28 are exact versions of Equations 9 and 19 with no damping. As a decelerating body passes through a given velocity (at $t=t_{o}$ ), it will match exactly the frequency of a constant velocity body (e.g., a free-to-oscillate wind tunnel model) with the same mass properties and in the same flow conditions. Prior to that time, the decelerating body will oscillate at a higher frequency, and later the frequency will decrease. This is consistent with the decrease in dynamic pressure for a decelerating body. 


\section{Application}

The Euler-Cauchy model describing the oscillation history of a decelerating object can be used in a number of applications. This section will illustrate some examples. First, the predictive capability will be shown against a simplified trajectory simulation to verify the model with a validated simulation code. Next the model will be fit to two high-fidelity data sets; a full 6-DoF simulation and ballistic range experimental data. These fits demonstrate the model can be used for preliminary parameter identification to determine constant coefficient estimates of nonlinear aerodynamics. Finally, a nondimensionalized version of the EulerCauchy equation will be used to design a dynamically scaled test article to match the dynamic behavior of a full-scale vehicle at a particular design point.

To solve Equation 21, the coefficients, $A$ and $\delta$ must be determined. Boundary conditions at the beginning and end points of the desired solution space must be provided. The assumption that velocity at time $t=0$ is very large permitted the moment equation to be cast in a form that has an analytical solution. However, it also requires that the equation be solved on a specific timeline. Equation 16 can be used to determine the times at which the body is traveling at specific velocities along this timeline. The following example illustrates this procedure.

\section{A. Euler-Cauchy Model of a Ballistic Range Case}

To demonstrate the behavior predicted by Equation 21, the oscillation amplitude history is calculated for a simple ballistic range case. The mass properties and aerodynamics will be those of a Mars Exploration Rover (MER) model (a $70^{\circ}$ degree sphere-cone blunt body with a truncated conic backshell ${ }^{3}$ ), tested at sea level conditions. To determine the coefficients of Equation 21, a timeline must be established that is consistent with the initial velocity assumption in Equation 17 that yielded the Euler-Cauchy form of the moment equation (Eq. 19). For such a timeline, it can be assumed that velocity is infinite at time $t=0$. This would imply infinite dynamic pressure (and therefore drag) and other violations of the physics of the problem, but these nonphysical conditions can be avoided by simply ensuring they are outside of the solution domain. Equation 16 can be used to determine the time after $t=0$, when the capsule reaches the first point in the solution space, $t\left(V=V_{1}\right)$, and any time up to the end boundary condition. For this example the initial boundary condition is Mach $=2.5\left(V_{1}=858 \mathrm{~m} / \mathrm{s}\right)$ and the end boundary condition is Mach $=1\left(V_{2}=343 \mathrm{~m} / \mathrm{s}\right)$. The times at which these conditions occur are determined by:

$$
t_{1}=\frac{2 m}{\rho S C_{A} V_{1}}, \quad t_{2}=\frac{2 m}{\rho S C_{A} V_{2}}
$$

A plot of Mach number versus time is shown in Figure 1. Substituting the vehicle-specific constants into Equations 22 and 24 yields values for $\mu$ and $\nu$. With $t_{1}$ established, Equation 21 and its first derivative at $t=t_{1}$,

$$
\dot{\alpha}_{1}=A \mu t_{1}^{\mu-1} \cos \left(\nu \ln t_{1}+\delta\right)-A \nu t_{1}^{\mu-1} \sin \left(\nu \ln t_{1}+\delta\right)=0
$$

can be rearranged to solve for the remaining constants, $A$ and $\delta$ :

$$
\begin{gathered}
\delta=\tan ^{-1}\left(\frac{\mu}{\nu}\right)-\nu \ln t_{1} \\
A=\frac{\alpha_{1}}{t_{1}^{\mu} \cos \left(\nu \ln t_{1}+\delta\right)}
\end{gathered}
$$

Table 1 lists the boundary conditions, freestream conditions, model mass properties and aerodynamic characteristics for Equation 21 for the MER ballistic range test. ${ }^{3}$ Figure 2 shows a plot of Equation 21 with all of the coefficients listed in Table 1 , from times $t=t_{1}$ to $t=t_{2}$. The different curves show the sensitivity of amplitude growth to the pitch damping coefficient, $C_{m_{q}}+C_{m_{\dot{\alpha}}}$. The coefficients for Equation 21 for each curve are listed in Table 2. The curves show the variation in oscillation frequency is due only to the reduction in dynamic pressure as the capsule decelerates. The amplitude growth decreases as the pitch damping coefficient varies from positive to negative values. A pitch damping value of -0.342 is required for no amplitude growth for this example. 
Table 1. Example Ballistic Range Parameters

\begin{tabular}{cc|cc|cc}
\multicolumn{2}{c|}{ Boundary Conditions } & \multicolumn{2}{c|}{ Range/Model Properties } & \multicolumn{2}{c}{ Aerodynamics } \\
\hline \hline$V_{1}$ & $858 \mathrm{~m} / \mathrm{s}$ & $m$ & $0.584 \mathrm{~kg}$ & $C_{m_{\alpha}}$ & $-0.09 \mathrm{rad}^{-1}$ \\
$V_{2}$ & $343 \mathrm{~m} / \mathrm{s}$ & $I$ & $1.55 \cdot 10^{-4} \mathrm{~kg} \cdot \mathrm{m}^{2}$ & $C_{A}$ & 1.58 \\
$\alpha_{0}$ & $5^{\circ}$ & $d$ & $0.07 \mathrm{~m}$ & $C_{m_{q}}+C_{m_{\dot{\alpha}}}$ & $+0.15,0,-0.15,-.342$ \\
$\dot{\alpha}_{0}$ & $0 \mathrm{rad} / \mathrm{s}$ & $S$ & $0.00385 \mathrm{~m}^{2}$ & $C_{N}$ & 0.0 \\
$t_{1}$ & $0.186 \mathrm{~s}$ & $\rho$ & $1.20 \mathrm{~kg} / \mathrm{m}^{3}$ & & \\
$t_{2}$ & $0.466 \mathrm{~s}$ & & & & \\
\hline
\end{tabular}

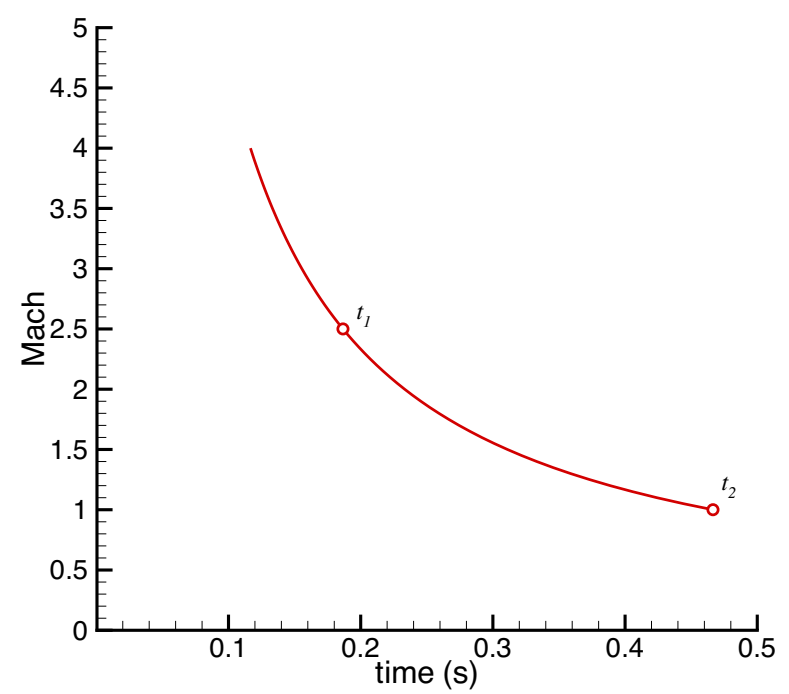

Figure 1. Mach number along Euler-Cauchy time domain, taken from Equation 29 with constants from Table 1.

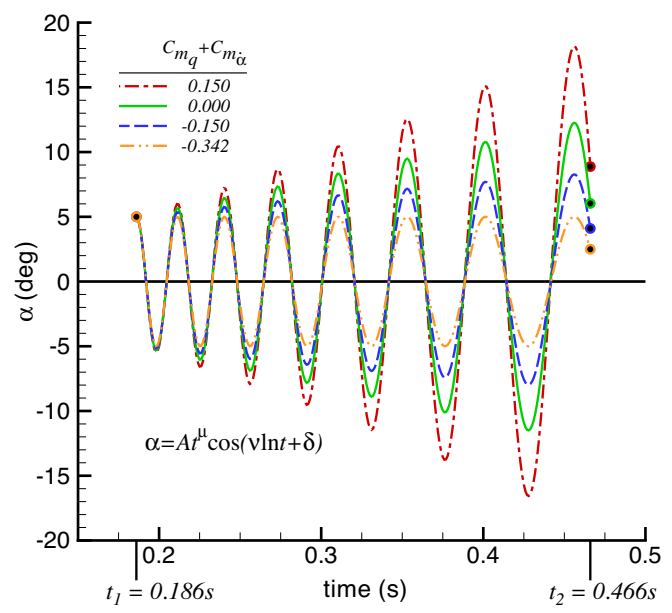

Figure 2. Variation of oscillation growth with pitch damping. See Table 2 
Table 2. Coefficients for Figure 2

\begin{tabular}{c|cccc}
$C_{m_{\dot{\alpha}}}$ & $A$ & $\mu$ & $\nu$ & $\delta$ \\
\hline \hline 0.150 & 56.009 & 1.438 & 49.060 & 0.752 \\
0.000 & 26.824 & 1.000 & 49.049 & 0.725 \\
-0.150 & 12.848 & 0.562 & 49.042 & 0.704 \\
-0.342 & 5.008 & 0.000 & 49.039 & 0.687 \\
\hline
\end{tabular}

To verify the angle-of-attack histories predicted by Equation 21, three of these cases were simulated in a 6 -DoF POST ${ }^{4}$ trajectory simulation and plotted against the analytic solutions. The aerodynamics used in the POST simulation were simplified to the constant values in Table 1, matching the assumptions made to derive the analytic solution. The trajectory simulation was run as a full 6 -DoF simulation but given initial conditions consistent with the planar equations and analytical solution (pure pitching motion and constant aerodynamic coefficients). Figure 3 compares the trajectory simulation results with the oscillation history predicted by Equation 21 for different constant values of the pitch damping coefficient. Note that there is almost perfect agreement between prediction and simulation, as would be expected given the simplifying assumptions used in the simulation.

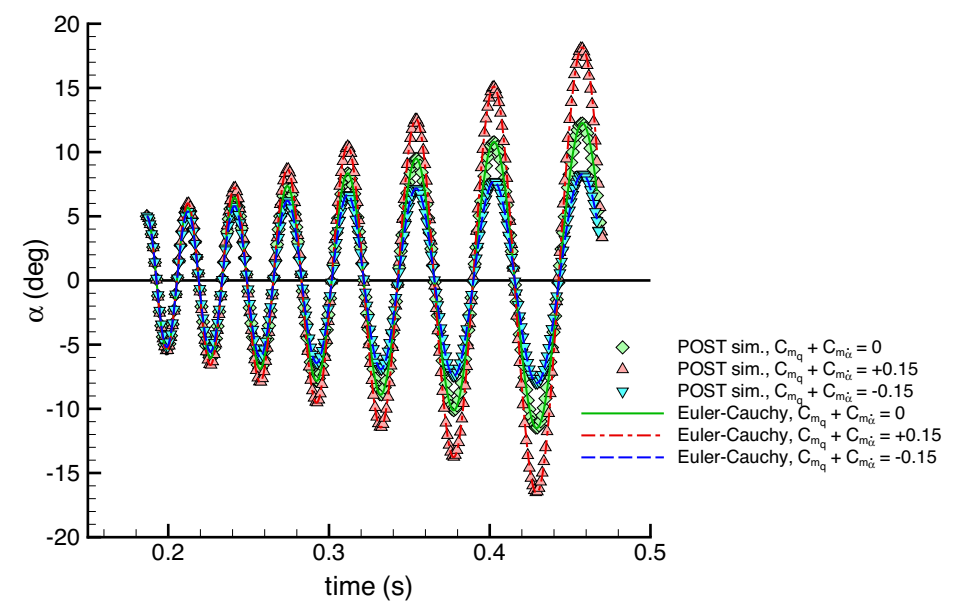

Figure 3. Comparison of Euler-Cauchy angle-of-attack solutions with simplified POST trajectory simulations.

\section{B. Curve Fits to Data}

It has been established that the Euler-Cauchy solution predicts the amplitude growth of a decelerating object with several simplifying assumptions. Now this model is fit to a nonlinear simulation and raw experimental data to illustrate how well the simple model agrees with these systems driven by more complex aerodynamics. The constant coefficients identified with the curve fitting process will be compared to the nonlinear values. This comparison will assess the applicability of the Euler-Cauchy model for modeling more realistic scenarios, both for the prediction of oscillation behavior, as well as first order parameter identification from experimental data.

Figures 4 and 6 show curve fits of Equation 21 through nonlinear simulation and experimental data. The constants, $A, \mu, \nu$ and $\delta$ in Equation 21 were extracted for both cases using a MATLAB curve fitting routine. Identifying the aerodynamic coefficients, $C_{A}, C_{m_{q}}+C_{m_{\dot{\alpha}}}$, and $C_{m_{\alpha}}$ from the Euler-Cauchy constants is a two step process. First, the axial force coefficient is determined evaluating Equation 15 over the solution domain: 


$$
C_{A}=\frac{2 m}{\rho S\left(t_{2}-t_{1}\right)}\left(\frac{1}{V_{2}}-\frac{1}{V_{1}}\right)
$$

Next, Equations 22 and 24 can be used to identify the pitch damping and static stability coefficients:

$$
\begin{gathered}
C_{m_{q}}+C_{m_{\dot{\alpha}}}=(\mu-1) \frac{4 I C_{A}}{m d^{2}} \\
C_{m_{\alpha}}=-\frac{\nu^{2} \pi \rho d I C_{A}^{2}}{8 m^{2}}
\end{gathered}
$$

Figure 4 shows a comparison of the Euler-Cauchy equation with a POST simulation of the MER ballistic range model. Even though the MER simulation uses nonlinear pitch damping, axial force and static stability data that varies significantly with angle-of-attack and Mach number, comparison is excellent. This shows that an "average" constant pitch damping value may replace a nonlinear pitch damping curve to provide a lower fidelity, but quantitative assessment of a capsule's dynamic stability.

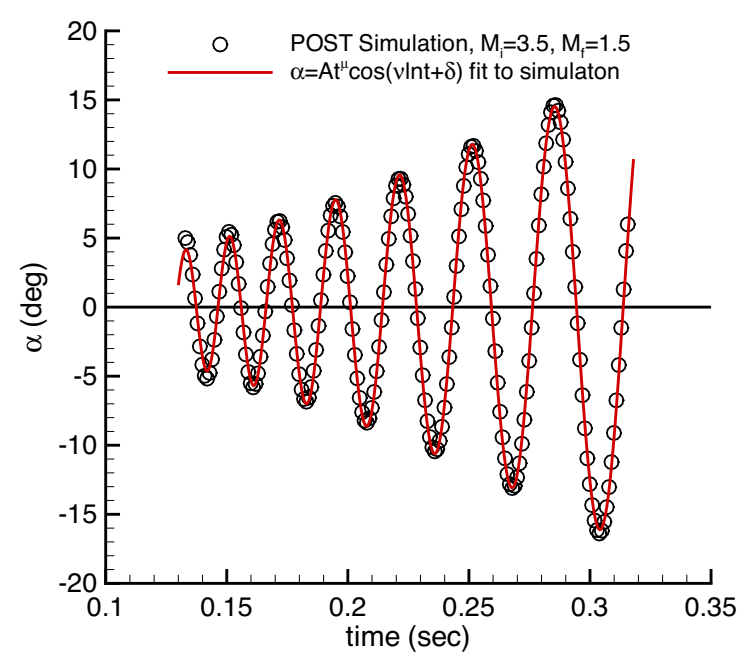

Figure 4. MATLAB curve fit through nonlinear simulation of MER ballistic range shot.

Figure 5 shows a comparison of the extracted constant coefficient values versus the aerodynamic coefficients in the MER aerodynamic database. ${ }^{5}$ The extracted constant coefficients fall within the nonlinear axial and pitch damping coefficients and appear to be reasonable mean values across the simulated Mach and angle-of-attack range. The most notable discrepancy is in static stability coefficient. This disagreement arises from the small angle assumption, made in the Euler-Cauchy development, that permits constant values of $C_{A}, C_{D}$ and $-C_{L_{\alpha}}$ to be interchanged (and $C_{N}=0$ ). Calculating $C_{A}$ from nonlinear simulation results using Equation 33 yields a value smaller than the mean of the nonlinear value of $C_{A}$ as it does not account for the fact that the capsule is oscillating and $C_{A}$ is directed at an angle relative to the direction of deceleration. This smaller axial force coefficient, when used in Equations 34 and 35 yields slightly smaller damping and static stability coefficients. As $C_{m_{\alpha}}$ is proportional to $C_{A}^{2}$ in Equation 35, small errors in axial force are amplified in the static stability. Larger oscillation amplitudes result in larger errors in the parameter identification. Overall, given all of the assumptions and simplifications of the Euler-Cauchy model, agreement is quite good and the model can be used for preliminary parameter identification.

Figure 6 shows a similar fit through raw data from the MER ballistic range test program. ${ }^{3}$ Refer back to Table 1 for model mass properties. Note that the raw angles measured in the ballistic range are not angle-of-attack and sideslip, but Euler angles in the range coordinate system. For these ballistic range shots where flight path angle is changing very little and oscillations are predominantly in one plane, the raw angles and the Euler-Cauchy prediction of oscillation growth may be compared for preliminary parameter identification. The coefficients identified from this curve fitting are also tabulated in Figure 6. Again, 


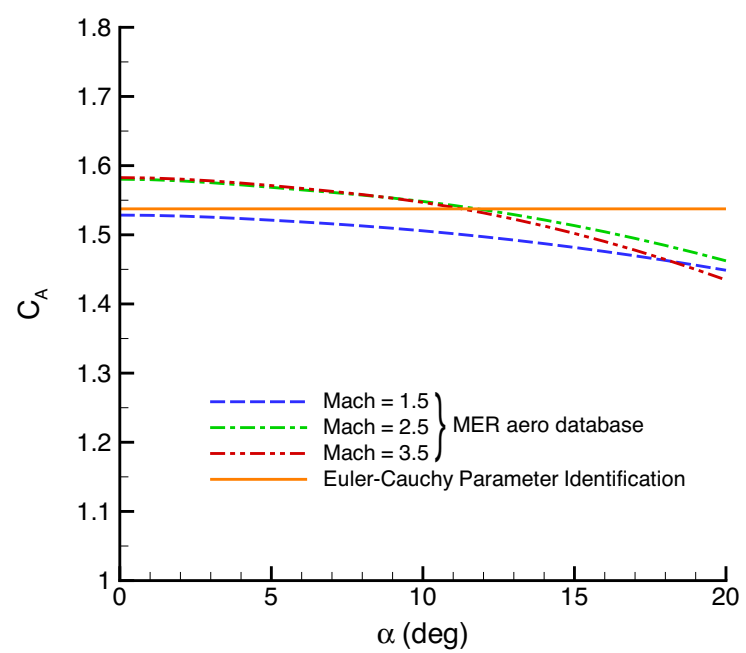

(a) Axial Force

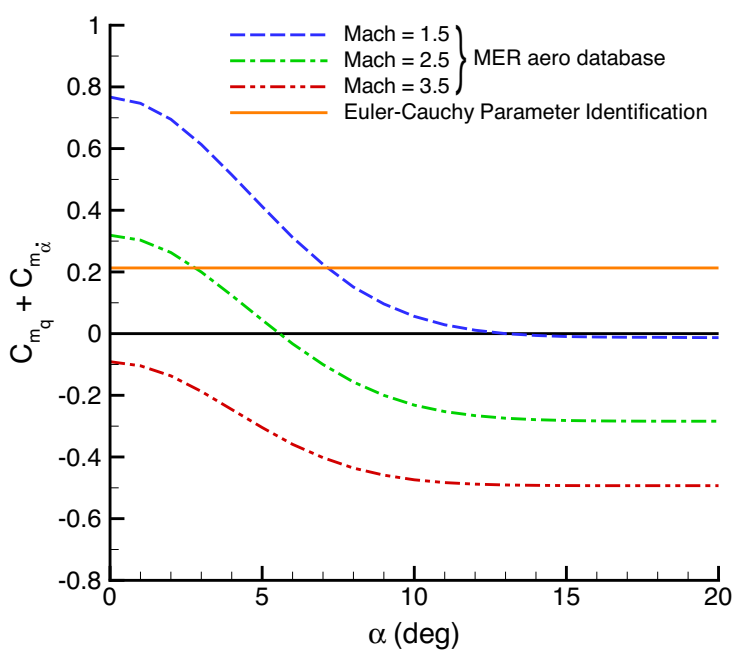

(b) Pitch Damping

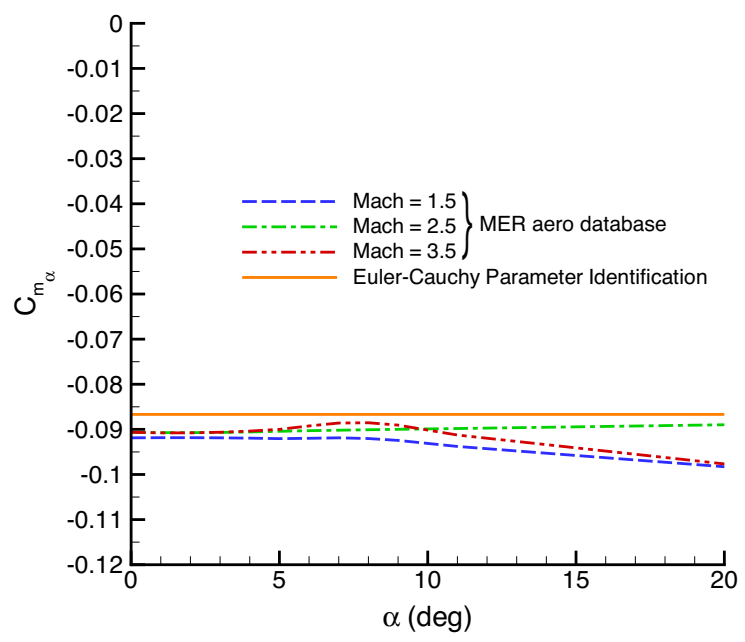

(c) Static Stability

Figure 5. Comparison of MER aerodynamics with coefficients obtained by fitting the Euler-Cauchy solution through a nonlinear simulation.

a close fit through the data suggests that this simple equation captures the observed behavior and the important parameters influencing the dynamics of ballistic range model flight at moderate angles-of-attack. The parameters extracted are also in reasonable agreement with the MER aerodynamic database.

Note that this method of parameter identification is only applicable to experimental data that is close to pure pitching motion in one plane. Three-dimensional motions such as coning or oscillations coupled with a significant spin rate have more complex oscillation histories in pitch and yaw, therefore requiring a full 6-DoF reconstruction. 


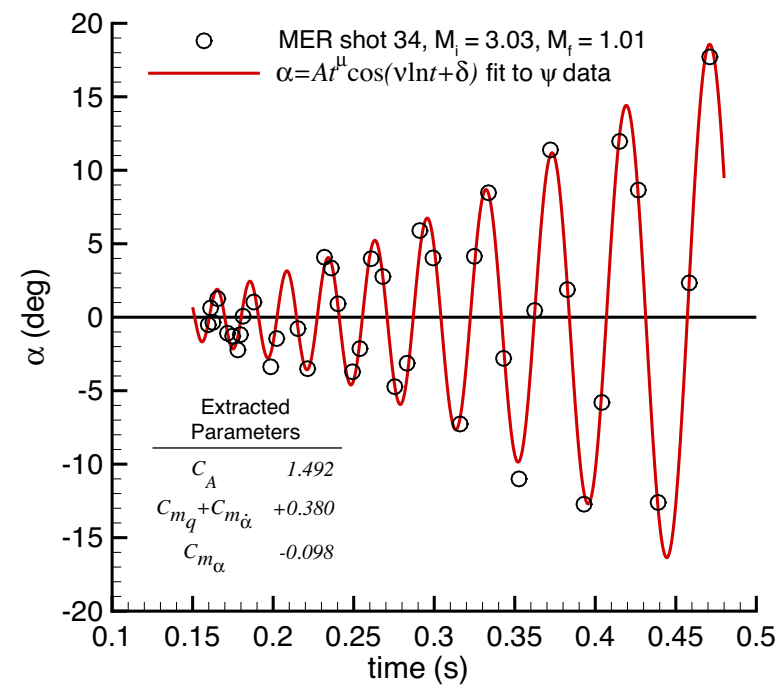

Figure 6. MATLAB curve fit through MER ballistic range experimental data (shot 34).

\section{Scaling Analysis}

The equations developed can be nondimensionalized for use in sizing scale models of flight vehicles for testing in ballistic range or scaled flight testing. Velocity and time are nondimensionalized as:

$$
\bar{V}=\frac{V}{s}=M, \quad \bar{t}=t \frac{s}{d}
$$

Where $s$ is the local sound speed. Time is nondimensionalized by the time it takes sound to travel a meaningful distance, here the reference length of the decelerating body. Substituting these relations into Equation 14 yields:

$$
\frac{\pi}{8} \frac{\rho d^{3}}{m} C_{A} M^{2}=\frac{d M}{d \bar{t}}
$$

Likewise, the moment equation for a decelerating body (Eq. 19) which describes the oscillatory behavior of a decelerating body can be nondimensionalized:

$$
\vec{t}^{2} \ddot{\bar{\alpha}}-\left(1+\frac{1}{2} \frac{m d^{2}}{I} \frac{C_{m_{\dot{\alpha}}}}{C_{A}}\right) \bar{t} \dot{\bar{\alpha}}-\frac{8}{\pi} \frac{m d^{2}}{I} \frac{m}{\rho d^{3}} \frac{C_{m_{\alpha}}}{C_{A}^{2}} \bar{\alpha}=0
$$

Recalling the reference area $S$ is equal to $\pi d^{2} / 4$, note that nondimensionalizing the moment equation does not change its form and Equations 38 and 19 are identical. Therefore Equation 21 describes the oscillation behavior versus nondimensionalized time as well:

$$
\bar{\alpha}=\alpha=A \bar{t}^{\mu} \cos (\nu \ln \bar{t}+\delta)
$$

Equations 37 and 38 contain the ratios, $\frac{m d^{2}}{I}$ and $\frac{m}{\rho d^{3}}$. To test a model that is a dynamically scaled to fullscale flight conditions in the nondimensional time frame, and assuming the same aerodynamic coefficients, all that is required is to match these two parameters. Here the ratios of mass, diameter, moment-of-inertia and density are defined:

$$
N_{m}=\frac{m_{\text {test }}}{m_{\text {flight }}}, \quad N_{d}=\frac{d_{\text {test }}}{d_{\text {flight }}}, \quad N_{I}=\frac{I_{\text {test }}}{I_{\text {flight }}}, \quad N_{\rho}=\frac{\rho_{\text {test }}}{\rho_{\text {flight }}}
$$

Then, the scaling laws for density and moment-of-inertia can be written: 


$$
\frac{N_{m}}{N_{\rho} N_{d}^{3}}=1 \quad \frac{N_{I}}{N_{m} N_{d}^{2}}=1
$$

By matching these relations for a full size vehicle and a scaled model, Equations 37 and 38 are identical. Mitcheltree $^{6}$ showed that these parameters are important for the dynamic scaling of bodies in subsonic, terminal descent testing. It is interesting that the same parameters fall out of equations for much higher velocity, where the Froude number and Mach number cannot be matched simultaneously. Here the assumptions regarding the trajectory (deceleration is significant or flight path angle is small) eliminate the requirement that Froude number be matched. Note that this analysis assumes identical aerodynamic coefficients and is limited to two degrees of freedom (angle-of-attack and velocity in the downstream direction). This analysis assumes constant density and does not account for trajectory effects caused by flight in a gravity field. However, the following example will show that dynamic scaling using Equation 38 can yield a useful scaled model design even when the constant density and trajectory flight-path-angle assumptions are violated.

The feasibility of a dynamically scaled test that replicates a segment of a high-altitude abort scenario was evaluated. To do this, a 6-DoF trajectory simulation of an Apollo capsule (a spherically blunted body with a conic backshell) was calculated from Mach $=4$ down to Mach $=1$. The static aerodynamics and capsule properties for this simulation were taken from the Apollo Block I aerodynamic database. ${ }^{7}$ The pitch damping coefficient, $C_{m_{q}}+C_{m_{\dot{\alpha}}}$, was set at a constant value of +0.15 . Then, a model was scaled to test at sea level, selecting a diameter, mass and moment of inertia about the pitch axis, using Equation 38 and the matching the ratios in Equation 40. For this sub-scale model, A 6-DoF trajectory was calculated, using identical aerodynamics for comparison. In the Apollo simulation this segment of the trajectory corresponds with a decrease in altitude from $26.7 \mathrm{~km}$ down to $17.6 \mathrm{~km}$. The scaled model was assumed to remain at sea level. Tables 3 and 4 list the freestream and model parameters for the full-scale and sub-scale configurations.

Table 3. Dynamic Scaling Freestream Conditions

\begin{tabular}{c|cccccc} 
& $\rho_{M=4}\left(\mathrm{~kg} / \mathrm{m}^{3}\right)$ & $\begin{array}{c}\rho_{M=2}\left(\mathrm{~kg} / \mathrm{m}^{3}\right) \\
\text { Scaled point }\end{array}$ & $\rho_{M=1}\left(\mathrm{~kg} / \mathrm{m}^{3}\right)$ & $\gamma_{M=4}(\mathrm{deg})$ & $\begin{array}{c}\gamma_{M=2}(\mathrm{deg}) \\
\text { Scaled point }\end{array}$ & $\gamma_{M=1}(\mathrm{deg})$ \\
Vehicle & & 0.067 & 0.130 & -28.9 & -35.1 & -51.4 \\
\hline \hline Apollo & 0.030 & 1.20 & 1.20 & 0.0 & -0.35 & -1.22 \\
Scaled & 1.20 & 1.20 & & \\
\hline
\end{tabular}

Table 4. Dynamic Scaling Model Parameters

\begin{tabular}{c|ccc} 
Vehicle & $m(\mathrm{~kg})$ & $d(\mathrm{~m})$ & $I\left(\mathrm{~kg} \cdot \mathrm{m}^{2}\right)$ \\
\hline \hline Apollo & 4989.5 & 3.91 & 5917.9 \\
Scaled & 5.04 & 0.15 & 0.00879 \\
\hline
\end{tabular}

Figure 7 shows a plot of angle-of-attack versus time for the full-scale and sub-scale trajectories. The scaled model, flying at sea level decelerates from Mach 4 to Mach 1 in less than a second, while the full-scale model takes approximately 27 seconds to decelerate. Against nondimensional time, however, the behavior of the vehicles is very similar.

Figure 8 shows a POST simulation of the oscillation history, against nondimensional time, of a ballistic range model, shot at sea level. The mass properties were selected to achieve a dynamically scaled model (satisfying the relations in Equation 40) of the full-scale Apollo capsule at Mach $=2.0$ along a full-scale trajectory. This history is plotted against a POST simulation of the corresponding full-scale Apollo capsule oscillation history. Even though the variation of density with time is not matched, and the flight-path-angle histories vary, the scaled case behaves in a manner very similar to the full-scale capsule versus nondimensional time. The nondimensional time that corresponds with Mach $=2.0$ for these cases is $\bar{t}=1135$. In Figure $8 \mathrm{a}$ at this time, both cases fall into very close agreement. The density variation along the Apollo trajectory results in a dilation of the nondimensional timeline. This dilation results in a mismatch between Mach number and 


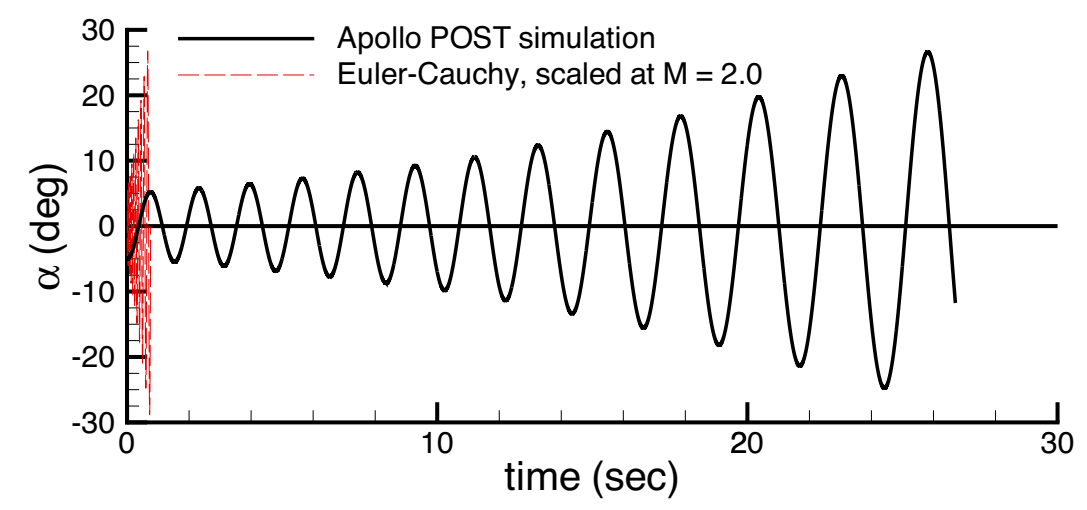

Figure 7. Comparison of full-scale Apollo flight with dynamically scaled model, $M_{i}=4.0, M_{f}=1.0$.
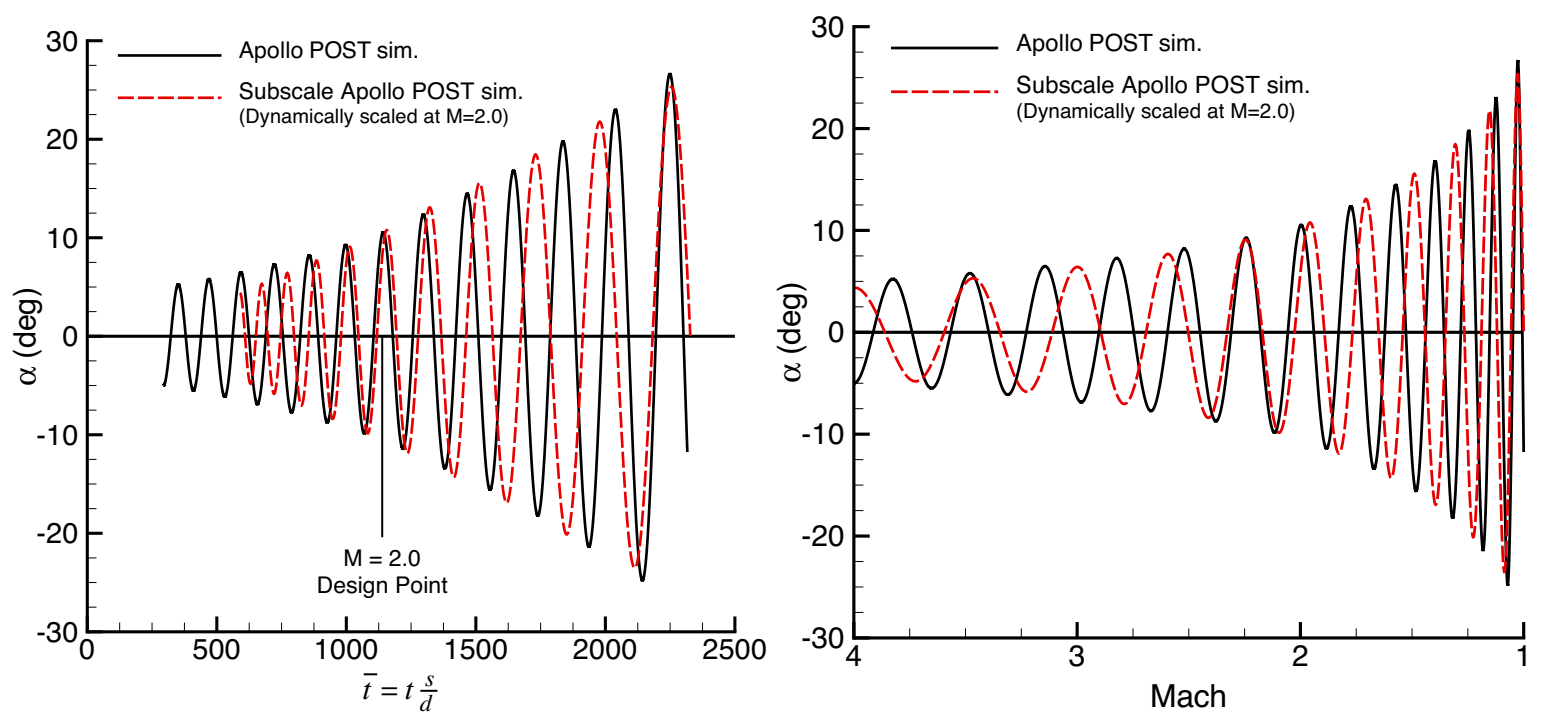

Figure 8. Oscillation growth of a full-scale Apollo and dynamically scaled capsules versus nondimensional time and velocity.

nondimensional time far from the scaling design point. The density variation also results in a deviation of the dynamic pressure history along the trajectory from that of the constant density sub-scale case. This accounts for the small deviation in amplitude growth and oscillation frequency between the two cases. Prior to the design point, the full-scale model is decelerating less rapidly and therefore the rate of oscillation amplitude growth is less. This is due to the lower density through which the capsule is traveling prior to the design point. After decelerating to speeds below Mach 2.0, the amplitude of the full-scale capsule oscillations grows slightly faster than the sub-scale model. This is consistent with the full-scale model flying deeper in atmosphere where density is greater than at the design point. Overall, these differences are small and the general behavior of the two vehicles is very similar. The oscillation frequencies and amplitude histories suggest that sub-scale testing of a dynamically scaled model can be used to obtain aerodynamics data from trajectories that very closely match those expected at full scale. 


\section{Conclusion}

The Euler-Cauchy equation has been shown to accurately describe the oscillation and deceleration characteristics of statically stable, low L/D objects with constant pitch damping. The simple analytic solution to the Euler-Cauchy equation provides a useful tool to design ballistic range test matrices. Using simple curve fitting routines, the function can be used to obtain useful preliminary assessments of ballistic range test data. The constant coefficients extracted from MER test data were shown to match very well with the full nonlinear MER aerodynamic database. The nondimensional form of the Euler-Cauchy equation provides a useful tool for dynamically scaling test models to match flight conditions. Even for a full-scale trajectory that has greatly varying density and flight-path-angle, this simple equation can provide mass properties for a scaled capsule that will closely approximate the full-scale flight behavior at different freestream conditions.

\section{References}

${ }^{1}$ Chapman, G. T. and Yates, L. A., "Limit Cycle Analysis of Blunt Entry Vehicles," AIAA 99-1022, January 1999.

${ }^{2}$ Kreyszig, E., Advanced Engineering Mathematics, John Wiley and Sons, Inc., New York, 1988.

${ }^{3}$ Schoenenberger, M., Hathaway, W., Yates, L., and Desai, P., "Ballistic Range Testing of the Mars Exploration Rover Entry Capsule," AIAA 2005-0055, January 2005.

${ }^{4}$ Brauer, G. L., Cornick, D. E., and Stevenson, R., "Capabilities and Applications of the Program to Optimize Simulated Trajectories (POST)," NASA CR 2770, February 1977.

${ }^{5}$ Schoenenberger, M., Cheatwood, F., and Desai, P., "Static Aerodynamics of the Mars Exploration Rover Entry Capsule," AIAA 2005-0056, January 2005.

${ }^{6}$ Mitcheltree, R. A., "Dynamic Scaling for Earth Based Testing of Mars Terminal Descent Dynamics," AIAA 2003-5391, 2003.

${ }^{7}$ Dodds, J. I., “Aerodynamic Data Manual for Project Apollo," SID 64- 174C, North American Aviation, Inc., 1966. 